\title{
The effect of task-relevant and task-irrelevant attentional cues and skill level on performance and knee kinematics of standing long jump
}

\author{
Ayoub Asadi ${ }^{1}$, Esmaeel Saemi²,*, Mahmoud Sheikh ${ }^{1}$, and Mehdi Takhtaei ${ }^{1}$ \\ ${ }^{I}$ Faculty of Sport Sciences, University of Tehran, Tehran, Iran; and ${ }^{2}$ Faculty of Sport Sciences, Shahid Chamran University \\ of Ahvaz, Ahvaz, Iran
}

Copyright: (C) 2018 A. Asadi et al. This is an open access article licensed under the Creative Commons Attribution License (http://creativecommons.org/licenses/by/4.0/).

\begin{abstract}
Background: Attention is one of the brain's most crucial cognitive functions playing a key role in performing motor skills. Objective: The purpose of the present study was to investigate the effect of task-relevant and task-irrelevant attentional cues and skill level on performance and knee kinematics of standing long jump. Methods: Fifteen skilled (volleyball and basketball players) and fifteen novices (aged 19 to 27 years) were recruited. The task was the standing long jump test which was executed 9 times under 3 conditions ( 3 trials per condition). Participants in the control condition did not receive any attentional instruction. In task-relevant attentional cues condition, participants were instructed to focus on their ongoing skill and avoid paying attention to matters unrelated to the task. In task-irrelevant attentional cues condition, participants were instructed to focus on sound and after each jump expressed what had been the musical content. Kinematic data of knee joint was recorded by an 8-camera motion analysis system. Results: The result of mixed ANOVA showed that both novice and skilled groups had significantly greater jump distance and smaller initial knee angle in task-irrelevant attentional cues condition compared to task-relevant attentional cues condition $(p=.001)$ and control condition $(p=.001)$. There were no significant differences between the control and task-relevant attentional cues condition $(p>.05)$. Conclusions: The result showed that focusing attention on taskirrelevant attentional cues improves standing long jump performance of both novice and skilled jumpers.
\end{abstract}

Keywords: attentional strategies, underlying mechanism, skilled, novice, motor control

\section{Introduction}

Defined as the level of awareness of the environment, attention is one of the brain's most crucial cognitive functions playing a key role in performing motor skills (Wulf, 2007). Given the importance of attention strategies for the enhanced performance of motor skills, researchers in motor behavior and sport psychology have made considerable efforts to understand what attentional styles lead to success in sport (Wulf, 2013). One of the subjects of interest to researchers in motor behavior in recent years has been the impact of various attention strategies on motor behavior (Wulf, 2013).

According to attentional focus literature, the coach or therapist can focus the performer's attention on particular aspects of the skill being performed just by

\footnotetext{
* Address for correspondence: Esmaeel Saemi, Department of Sport Psychology, Faculty of Sport Sciences, Shahid Chamran University of Ahvaz, Golestan Blvd., Ahvaz, 61357-83151 Iran. E-mail: e.saemi@scu.ac.ir
}

changing the words and phrases of their verbal instructions, thus influencing their motor performance and learning in different ways (Beilock, Carr, Macmahon, \& Starkes, 2002; Wulf, 2013). Studies on the focus of attention have sought to define attention instructions from two perspectives. The first perspective developed by Wulf and her students studies the focus of attention from both internal and external points of view (Wulf, 2013). Most of the studies conducted using such a perspective have demonstrated the advantage of the external focus of attention (Saemi, Abdoli, Farsi, \& Sanjari, 2017).

The second perspective developed by Beilock et al. (2002) mainly concerns the effect of attention on motor performance focusing on task-relevant and taskirrelevant aspects attentional cues (Beilock, Bertenthal, McCoy, \& Carr, 2004; Beilock \& Carr, 2001; Castaneda \& Gray, 2007; Gray, 2004). Under conditions of task-relevant attention, the performer consciously focuses his/her attention on the aspects related to the task being performed (e.g., the movement of the hands 
or the ball during a basketball free throw shot). Under conditions of task-irrelevant attention, the performer focuses his/her attention on environmental aspects unrelated to the task being performed (e.g., the spectators' voice during a basketball free throw).

Based on the findings of such studies, skilled and unskilled individuals demonstrate their best performance under task-irrelevant conditions in comparison with task-relevant attentional cues respectively (Beilock et al., 2004, 2002). The explicit monitoring theory has been developed based on these findings. According to this theory, beginners tend to consciously control and evaluate their newly acquired motor skills, while performing such skills among their skilled counterparts involves a smaller degree of conscious control processes and a higher degree of automatic and nonconscious ones (Beilock et al., 2004, 2002). In other words, these studies show that athletes with different skill levels (beginners as opposed to more skilled athletes) are differently affected by focus cues. Thus, skilled individuals and beginners show their best performance under task-irrelevant conditions and task-relevant attentional cues respectively. Other studies have also supported this hypothesis (Beilock et al., 2004, 2002; Castaneda \& Gray, 2007; Gray, 2004).

The direct evaluation hypothesis shows that the advantages associated with different attention strategies would vary based on the individual's skill level (Beilock et al., 2004, 2002). Castaneda and Gray (2007) carried out an experiment to study the interaction between internal and external attention instructions as well as the interaction between task-relevant and task-irrelevant attentional cues. In this experiments, the subjects (a group of baseball beginners and skilled players) were asked to perform a primary task (simulating a baseball swing) simultaneously with a secondary task related to the primary task (paying attention to the hands or the baseball club's movement at the moment of hearing a particular sound) or a secondary task unrelated to the primary task (paying attention to the frequency of a particular sound or the ball's direction in relation to the position of an explorer). Their findings varied based on the level of the subjects' skill. Thus, skilled subjects demonstrated significantly better performance under conditions of task-irrelevant attentional cues, while the beginners had better performance under conditions of task-relevant attentional cues regardless of whether it was internal or external (Castaneda \& Gray, 2007).

In more recent studies, kinematic variables of the human body are used to accurately evaluate performance. However, research on this topic remains limited. Ducharme, Wu, Lim, Porter, and Geraldo (2016) investigated the focus of attention effect on kinetic and kinematic variables associated with the standing long jump. The result showed that external focus caused greater jump distance and less projection angle rather than baseline and internal condition. But in kinetic measures (peak force and impulse) no significant differences among conditions were found. They suggested that external focus producing a more optimal projection angle that leads to a better jump (Ducharme et al., 2016). Although several studies have been conducted on task-relevant and task-irrelevant attentional cues, few studies have examined the effect of task-relevant and task-irrelevant attentional instructions on attention performance based on kinematic variables. In view of the fact that the underlying mechanisms of task-relevant and task-irrelevant attentional strategies have not been examined in previous studies, and to have a more adequate understanding of the mechanisms involved in the effectiveness of such strategies and their different effects particularly among people with varying skill levels, the present study seems to be warranted. Therefore, the aim of the study was to investigate the effect of task-relevant and task-irrelevant attentional cues and skill level on performance and knee kinematics of standing long jump.

\section{Methods}

\section{Participants}

Thirty male undergraduate students with the mean age of $23 \pm 4$ years in two groups of skilled (basketball and volleyball players) and novice (non-athletes) subjects were recruited. A priori power analysis showed that 30 participants would be sufficient to identify a significant effect of the two independent variables with a repeated measurements between-within-subject design with a power $(1-\beta)$ of .82, effect size of .25 , and an $\alpha$ of .05 (Faul \& Erdfelder, 1992). The experimental methods were approved by the ethical committee of the university. The participants were not aware of the objectives of the research and had signed an informed consent form.

\section{Task and instruments}

A scaled $1 \times 4$ rubber layer was placed on a hard surface to measure the standing long jump distance. The starting point was marked by a white line and the participants started their task from the behind this line. Following every jump, the distance covered from the starting point of jump to the trace on landing heel was recorded as the standing long jump performance. The kinematic data was recorded by 8 infra-red cameras (Osprey, Motion Analysis Corporation, Santa Rosa, CA, USA) capable of 240 frame per second using Cortex software (Version 2.6; Motion Analysis Corporation, Santa Rosa, CA, USA). For assuring proper data 
without noise, the testing environment was calibrated prior to each test session. The reflective markers with $14 \mathrm{~mm}$ diameter were placed on the anatomical locations including greater trochanter of hip, lateral condyle of tibia and lateral edge of mid-calf to measure kinematic variables of knee flexion. The jump angle (initial knee angle) was defined as the knee joint angle during the time of lifting the feet.

\section{Kinematic analysis}

Following the collection of kinematic data, the process of smoothing using cubic spline for each marker was performed. Then, the numeric data were prepared in Microsoft Excel (Version 2016; Microsoft, Redmond, WA, USA) and kinematic analysis was performed for knee joint.

\section{Procedures}

The participants performed a submaximal warm-up preparation for 5 minutes. They remained seated while listening to the instructions about how to take position to perform the task correctly. For the purpose of avoiding the first order effect, all the participants performed the task similar to the control condition, then the order of performance of task-relevant and task irrelevant conditions were counterbalanced across the participants. In the control condition, the instruction was based on "jump to the best of ability", in the task-relevant attentional focus condition, the instruction based on "jump to the best of ability" and "focusing on the performing of the task". In the task-irrelevant attentional focus condition, the jump was performed based on "the best of ability" and the instruction included "focusing on a sound which was played at the lab". The sound was a whistle at the same time as the performing the task at the lab. All the participants performed three trials in each stage and a total of 9 trials with a 2-minute rest between trials and 2 minutes' rest between each condition. During this time, the participants listened to attentional instructions. All the testing procedures were completed in one day and lasted 30 minutes.

\section{Statistical analyses}

SPSS (Version 18.0; SPSS, Chicago, IL, USA) was used for analyzing the standing long jump performance and kinematic maximum knee flexion data. Data were analyzed via a 2 (groups: skilled vs. novice) $\times 3$ (conditions: task-relevant, task irrelevant, and no focus) two-way mixed model analysis of variance (ANOVAs) with repeated measures on the last factor. Effect size was reported as $\eta^{2}$ (small $=.01$, average $=.06$, and large $=.14$ ) for Mixed ANOVA (Cohen, 1988). For all the statistical tests the significance was stated at level of $\alpha=.05$.

\section{Results}

\section{Performance}

Mauchly's test showed that the sphericity assumption had not been violated $(p=.81)$ and the result of mixed ANOVA-repeated measure showed a significant main effect for the groups $(F(1,28)=7.28, p=.001$, $\left.\eta^{2}=.99\right)$. The main effect of conditions was also significant $\left(F(2,56)=29.91, p=.001, \eta^{2}=.51\right)$. The result of Bonferroni test indicated that the task-irrelevant attentional cues $(226.36 \pm 14.64 \mathrm{~cm})$ was significantly superior to the control $(219.38 \pm 13.31 \mathrm{~cm})$ and taskrelevant $(215.97 \pm 15.15 \mathrm{~cm})$ conditions $(p=.01)$. However, there was no significant differences between the control and task-relevant focus attention $(p=.12)$. No significant interaction was found between the condition and the groups $(F(2,56)=1.64, p=.20$, $\eta^{2}=.05$; Figure 1).

\section{Kinematics}

Mauchly's test result of joint angle confirmed the sphericity condition $(p>.05)$ and the result of mixed ANOVA-repeated measure showed significant main effect for the groups $(F(1,28)=2.77, p=.001$, $\left.\eta^{2}=.99\right)$, also the main effect of attentional condition was significant $\left(F(2,56)=12.28, p=.001, \eta^{2}=.30\right)$. The result of Bonferroni test indicated that the taskirrelevant condition $\left(45.31 \pm 1.63^{\circ}\right)$ had significantly less initial knee angle compared to the other conditions $(p=.02)$. However, there was no significant differences between the control $\left(46.70 \pm 2.59^{\circ}\right)$ and task-relevant $\left(47.54 \pm 1.92^{\circ}\right)$ focus attention $(p=.23)$. No significant interaction was found between the condition and the groups $\left(F(2,56)=1.26, p=.28, \eta^{2}=.04\right.$; Figure 2$)$.

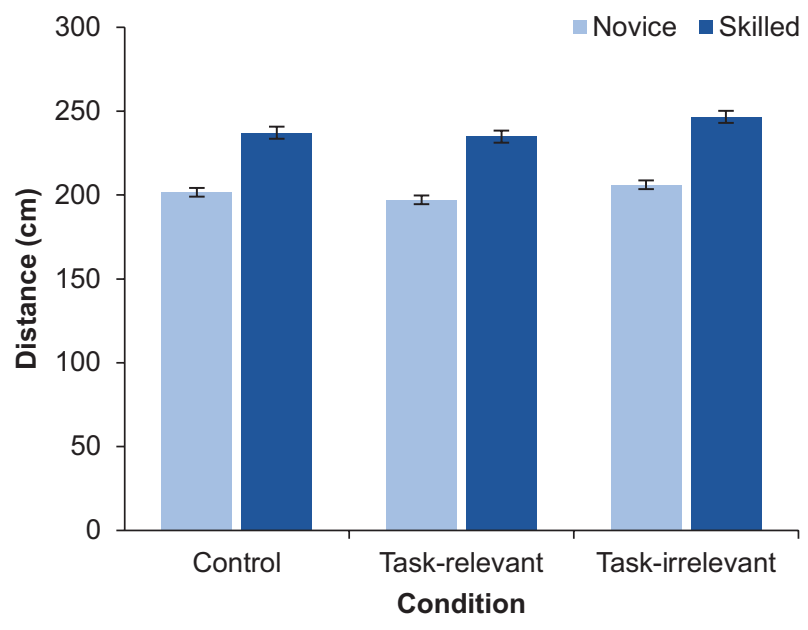

Figure 1. Standing long jump outcome measures (mean) with different attentional cues in novice and skilled participants. Error bars represent standard deviation. 


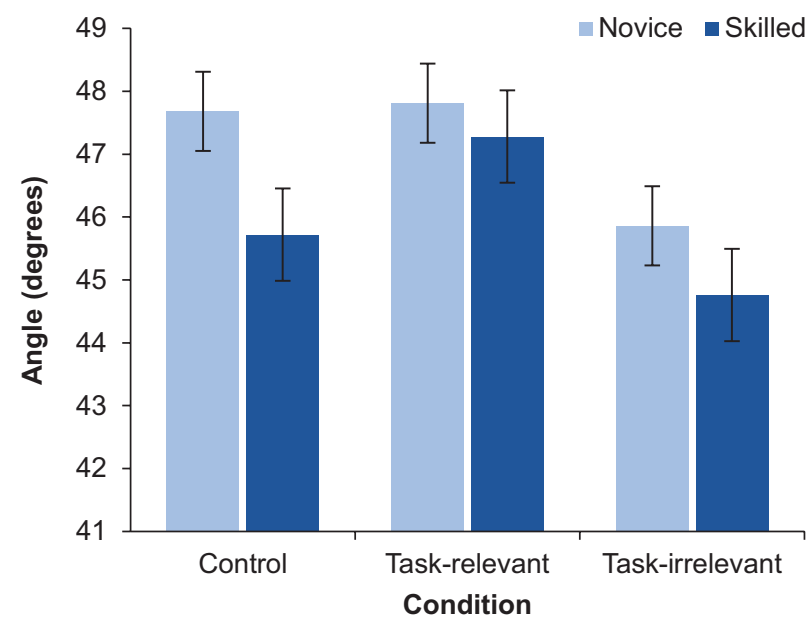

Figure 2. Initial knee angle (mean) with different attentional cues in novice and skilled participants. Error bars represent standard deviation.

\section{Discussion}

This study is an attempt to examine the effects of taskrelevant and task-irrelevant focus cues on the performance and knee kinematics of novice and skilled participants in standing long jump. The results showed no significant difference between the two groups in terms of optimum focus cues in performance, jump length and kinematics (initial knee angle). Thus, the novice and skilled participants in the task-irrelevant attention condition showed a higher jump length and lower initial knee angle compared with both the control and the task-relevant condition. The above results, however, are not compatible with those obtained by Beilock and Carr (2001), Beilock et al. (2002), Beilock et al. (2004), Gray (2004), and Castaneda and Gray (2007) in performance.

According to the explicit monitoring theory (Beilock \& Carr, 2001), beginners and professionals will react differently to different types of attention instruction (task-relevant or task-irrelevant attention instructions). Thus, beginners will show enhanced performance under conditions of task-relevant attention, while professionals show this enhanced performance under conditions of task-irrelevant attention (Beilock et al., 2002).

According to our findings, however, the data related to the performance of skilled jumpers is compatible with the predictions made based on the explicit monitoring theory and other research findings (Beilock et al., 2004, 2002; Beilock \& Carr, 2001; Castaneda \& Gray, 2007; Gray, 2004). But the data related to the performance of beginners is not compatible with the predictions made based on that hypothesis. This incompatibility may be partially caused by the secondary tasks used in the present study. In this study the subjects were asked to perform a long jump under conditions of task-relevant attentional cues. This focus on the jumping skill may have caused the subjects to adopt an internal attention instruction, leading to a decline in performance as the constrained action hypothesis indicates (Wulf, McNevin, \& Shea, 2001). In other words, lower performance of novices under conditions of task-relevant attention can be explained by the fact that task-irrelevant attentional instructions have created an external attention instruction. On the other hand, the use of task-irrelevant attentional instructions may prevent the adoption of internal attention instructions, thus developing automatic control processes in the motor system of beginners, improving their motor performance (Wulf et al, 2001).

In terms of kinematics, the results of this study indicate that maximum flexion on the knee joints before the take-off phase (initial knee angle) is lower in both novices and experts under conditions of task-irrelevant attentional cues. Theoretically, the optimum initial knee angle for jumping is an angle of between $45^{\circ}$ to $50^{\circ}$ (Alexander, 1990). However, other studies have shown that angles lower than $45^{\circ}$ increase the jumping length (Aguado, Izquierdo, \& Montesinos, 1997; Wakai $\&$ Linthorne, 2005), and that an increase in the angle reduces velocity in standing long jump. Thus, there is a negative correlation between initial knee angle and velocity of standing long jump (Linthorne, Guzman, \& Bridget, 2005).

According to the present study, therefore, the participants in the task-irrelevant attentional cues have shown lower maximum flexion compared to those in the control and the task-relevant attentional cues condition. Their enhanced jumping performance is apparently caused by a lower jumping performance. This finding partially supports previous findings by Ducharme et al. (2016) that found the participants in the external attention condition demonstrated their best performance at an angle of $45^{\circ}$, significantly lower than the optimum angle for the control and the internal attention condition. This caused the participants in the external attention condition to demonstrate a significantly higher jumping length compared with the other condition (Ducharme et al., 2016).

Focus instructions and its related cues may have developed an internal focus of attention. Thus, according to the constrained action hypothesis, the subject has to simultaneously focus his/her attention on the limbs involved in performing the motor skill and the effects of their movements on the environment and their outcome. As a result, the pressure associated with performing the move intensifies, causing higherlevel nervous centers to become constantly involved 
in managing the limbs. This increases attention needs, causing disruptions in the automatic control of the movements through freezing degree of freedom, inappropriate activation of muscular chords, attention limitations, and negative coordination among joints. In contrast, focusing on task-irrelevant attentional cues enhances external attention. According to the constrained action hypothesis, this type of attention reduces the number of processes involved, leading to reduced attention needs, automatic activation of the motor system through degrees of freedom, efficient patterns of muscular chords activation, enhanced attention capacity for action, better coordination among the joints (Wulf, 2013; Wulf et al., 2001).

Given the fact that no significant difference was observed between beginners and skilled participants in terms of both kinematics and performance regarding optimum focus cues, the findings of this study do not support the explicit evaluation hypothesis, somehow supporting the constrained action hypothesis (Wulf et al., 2001). Inconsistencies in the findings of previous studies like that of Beilock et al. (2002) and subsequent studies may have been caused by differences in methodology rather than differences in theoretical framework. In her review article, Wulf has pointed out to some of these factors including ambiguous, complex and multidimensional attention instructions and different ways of using simultaneous tasks to direct the subjects' attention toward the task-relevant and task-irrelevant aspects (Wulf, 2013).

Review of the relevant literature shows that the present study has been the first study in which taskrelevant and task-irrelevant attentional focus cues in knee joints have been examined. The results showed that paying attention to external task-irrelevant cues enhances performance and explosiveness in standing long jump. Given the fact that long jump is a complex move involving multiple muscles and requiring a high level of coordination, it is suggested that future studies should examine musculature involved such as the thigh and the lower leg to study the effects of various focus cues on coordination among the joints. For the purposes of the present study, skilled participants were those who had been playing basketball or volleyballs in university teams. Although we classified these individuals as skilled participants, it is suggested that more experienced people should be used as experiment subjects. The level of the subjects' automaticity varies based on their skill level, generating different responses to different attention instructions.

\section{Conclusions}

Furthermore, the results suggest that coaches should use those attention instructions directing the subjects' attention away from the body and the task being performed for teaching motor skills (particularly those requiring peak force) and for maximizing performance in such skills.

\section{Acknowledgments}

We gratefully acknowledge the volunteers involved in this study.

\section{Conflict of interest}

There were no conflicts of interest.

\section{References}

Aguado, X., Izquierdo, M., \& Montesinos, J. L. (1997). Kinematic and kinetic factors related to the standing long jump performance. Journal of Human Movement Studies, 32, 157-170.

Alexander, R. M. (1990). Optimum take-off techniques for high and long jumps. Philosophical Transactions: Biological Sciences, 329(1252), 3-10.

Beilock, S. L., Bertenthal, B. I., McCoy, A. M., \& Carr, T. H. (2004). Haste does not always make waste: Expertise, direction of attention, and speed versus accuracy in performing sensorimotor skills. Psychonomic Bulletin \& Review, 11, 373-379.

Beilock, S. L., \& Carr, T. H. (2001). On the fragility of skilled performance: What governs choking under pressure? Journal of Experimental Psychology: General, 130, 701-725.

Beilock, S. L., Carr, T. H., Macmahon, C., \& Starkes, J. L. (2002). When paying attention becomes counterproductive: Impact of divided versus skill-focused attention on novice and experienced performance of sensorimotor skills. Journal of Experimental Psychology: Applied, 8, 6-16.

Castaneda, B., \& Gray, R. (2007). Effects of focus of attention on baseball batting performance in players of differing skill levels. Journal of Sport and Exercise Psychology, 29, 60-77.

Cohen, J. (1988). Statistical power analysis for the behavioral sciences (2nd ed.). Hillsdale, NJ: Erlbaum.

Ducharme, S. W., Wu, W. F., Lim, K., Porter, J. M., \& Geraldo, F. (2016). Standing long jump performance with an external focus of attention is improved as a result of a more effective projection angle. Journal of Strength and Conditioning Research, 30, 276-281.

Faul, F., \& Erdfelder, E. (1992). GPOWER: A priori, posthoc, and compromise power analyses for MS-DOS [Computer software]. Bonn, Germany: Bonn University. 
Gray, R. (2004). Attending to the execution of a complex sensorimotor skill: Expertise differences, choking, and slumps. Journal of Experimental Psychology: Applied, 10, 42-54.

Linthorne, N. P., Guzman, M. S., \& Bridgett, L. A. (2005). Optimum take-off angle in the long jump. Journal of Sports Sciences, 23, 703-712.

Saemi, E., Abdoli, B., Farsi, A., \& Sanjari, M. A. (2017). The interaction of external/internal and relevant/irrelevant attentional focus on skilled performance: The mediation role of visual information. Medicina Dello Sport, 70, 419-429.
Wakai, M., \& Linthorne, N. P. (2005). Optimum take-off angle in the standing long jump. Human Movement Science, 24, 81-96.

Wulf, G. (2007). Attention and motor skill learning. Champaign, IL: Human Kinetics.

Wulf, G. (2013). Attentional focus and motor learning: A review of 15 years. International Review of Sport and Exercise Psychology, 6, 77-104.

Wulf, G., McNevin, N., \& Shea, C. H. (2001). The automaticity of complex motor skill learning as a function of attentional focus. Quarterly Journal of Experimental Psychology: Section A, 54, 1143-1154. 